\title{
Dipeptidyl Peptidase IV Inhibition Improves Impaired Glucose Tolerance in High-Fat Diet-Fed Rats: Study Using a Fischer 344 Rat Substrain Deficient in Its Enzyme Activity
}

\author{
Hironobu Mitani ${ }^{1} *$, Misato Takimoto ${ }^{1}$, Thomas E. Hughes ${ }^{2}$ and Masaaki Kimura ${ }^{1}$ \\ ${ }^{1}$ Research Division, Tsukuba Research Institute, Novartis Pharma K.K., Ohkubo 8, Tsukuba 300-2611, Japan \\ ${ }^{2}$ Novartis Institute for Biomedical Research, 556 Morris Avenue, Summit, New Jersey 07901, USA
}

Received September 18, 2001 Accepted January 16, 2002

\begin{abstract}
This study was performed to determine the effects of a high-fat diet on glucose metabolism after an oral glucose challenge in high-fat diet-fed dipeptidyl peptidase IV (DPP-IV) positive $(+)$ and deficient (-) Fischer 344 (F344) rats and the effects of novel DPP-IV inhibitor NVP-DPP728 (1-\{2-[(5-cyanopyridin-2-yl)amino]ethylamino\}acetyl-2-cyano-(S)-pyrrolidine monohydrochloride salt) on glucose tolerance in high-fat diet-fed F344 rats. In DPP-IV(+) rats, a high-fat diet load caused impaired glucose tolerance, such as increases of plasma insulin and blood glucose concentrations after oral glucose challenge, compared with a standard chow-fed group. In contrast, no marked change in glucose tolerance was induced by the high-fat diet in DPP-IV(-) rats. Blood glucose concentrations in DPP-IV $(-)$ rats after glucose challenge were significantly lower than in DPP-IV $(+)$ rats under high-fat diet load conditions. In standard chow and high-fat dietfed DPP-IV $(+)$ rats, NVP-DPP728 significantly suppressed glucose excursions after glucose challenge by inhibiting the plasma DPP-IV activity, associated with the stimulation of early insulin secretion. NVPDPP728 did not affect glucose tolerance in DPP-IV(-) rats under both conditions. These results indicate that the amelioration of glucose tolerance by NVP-DPP728 in DPP-IV $(+)$ rats was directly due to the inhibition of plasma DPP-IV activity, which might be via the subsequent increase in endogenous incretin action.
\end{abstract}

Keywords: Dipeptidyl peptidase IV, Fischer 344 rat, Glucose tolerance, High-fat diet, Diabetes

The truncated form of glucagon-like peptide-1 (GLP-1), a 30 amino acid polypeptide, is known to be one of the important insulin-releasing hormones (incretins) and is postprandially released by the enteroglucagon-producing L cells in the lower gut, i.e., the ileum $(1-3)$. GLP-1 regulates not only blood glucose via stimulation of glucosedependent insulin secretion, but also inhibition of gastric emptying and inhibition of glucagon secretion $(1-3)$. In addition, GLP-1 may also regulate food intake in the central nervous system and glycogen synthesis in adipose tissue and skeletal muscle $(1-3)$. Importantly, it has been demonstrated that the intravenous or subcutaneous administration of GLP-1 is highly efficacious in patients with diabetes, normalizing hyperglycemia in moderate-to-severe type 2 diabetes subjects $(4-7)$. This raises the possibility of its use as a therapeutic agent. Recent studies have shown that disruption of the GLP-1 receptor gene results in fasting hyperglycemia and abnormal glycemic excursions after

*Corresponding author. FAX: +81-298-65-2385

E-mail: hironobu.mitani@pharma.novartis.com glucose challenge together with reduced levels of glucosestimulated insulin (8), emphasizing the essential role of GLP-1 in the control of blood glucose level.

GLP-1 is metabolically unstable, having a plasma halflife of only $1-2 \mathrm{~min}$ in vivo $(9,10)$. It has been suggested that the circulating level of active GLP-1 in humans and rats $(9,11)$ is primarily regulated by dipeptidyl peptidase IV (EC 3.4.14.5; DPP-IV), a serine aminopeptidase, which has been identified as the enzyme that inactivates GLP-1 from active GLP-1(7-36) amide to GLP-1 $(9-36)$ amide by cleaving the $\mathrm{N}$-terminal His-Ala dipeptide fragment (9). The difficulty of the development of GLP-1 as a therapeutic agent has been due to the lack of oral bioavailability of GLP-1, and its rapid degradation to GLP-1 $(9-36)$ which can antagonize the effects of GLP-1(7 - 36) amide (12).

Recent studies in anesthetized pigs have demonstrated that acute DPP-IV inhibition by the specific DPP-IV inhibitor, valine-pyrrolidide, potentiated the insulinotropic effect of intravenous administered GLP-1 by inhibiting the $\mathrm{NH}_{2}$-terminal degradation of GLP-1 (13). Acute oral administration of DPP-IV inhibitors have augmented insulin res- 
ponses to oral glucose challenge and enhanced glucose clearance in insulin resistant obese Zucker rats $(14,15)$. These results indicate that DPP-IV inhibitors have potential as new therapeutic agents for type 2 diabetes $(16,17)$.

Watanabe et al. (18) have shown that Japanese Fischer 344 (F344) rats lack DPP-IV enzyme activity. DPP-IV deficient $(-)$ rats contain mRNA transcripts for DPP-IV but reduced levels of DPP-IV protein due to the translation of abnormal isoforms that fail to be processed to the biologically active mature glycosylated enzyme $(19,20)$. These rats have reduced N-terminal degradation of GLP-1 by DPP-IV following infusion of GLP-1 (9), and have been used in studies on the role of DPP-IV/CD26 in the immune system including $\mathrm{T}$-cell activation, transplantation, and arthritis $(21-24)$. However, not much is known about the glucose metabolism after oral glucose challenge in DPPIV(-) F344 rats especially in diabetic conditions, e.g., high-fat diet load. Also, the effect of long-term inhibition of DPP-IV activity by a DPP-IV inhibitor on glucose excursion remains unclear. Therefore, DPP-IV(-) rats are thought to be a useful model for investigating the effect of longterm depletion of DPP-IV activity in diabetic conditions.

First, the present study was designed to confirm the deficiency of DPP-IV in Japanese F344 rats. Secondly, the glucose metabolism after oral glucose challenge in chronically cannulated standard chow and high-fat diet-fed DPP-IV positive $(+)$ and $(-)$ F344 rats was investigated, and thirdly, the effects of the novel potent DPP-IV inhibitor, NVPDPP728 $(15,25)$, on the glucose excursion after an oral glucose load in chow and high-fat diet-fed DPP-IV $(+)$ and (-) F344 rats were determined.

\section{MATERIALS AND METHODS}

\section{Materials}

NVP-DPP728 (1-\{2-[(5-cyanopyridin-2-yl)amino]ethylamino acetyl-2-cyano-(S)-pyrrolidine monohydrochloride salt) was synthesized in Novartis Pharma (Summit, NJ, USA) (25). Diprotin A, a competitive substrate of DPP-IV (26), was purchased from Calbiochem-Novabiochem (Laufelfingen, Switzerland).

\section{Animals and surgery}

Fischer 344 (F344) DPP-IV(+) and F344 DPP-IV(-) rats were purchased from Clea Japan (Tokyo) and Charles River Japan (Osaka) respectively. Sprague-Dawley (SD) rats were purchased from Charles River Japan. Rats were given standard rodent chow (CE-2, Clea Japan) and water ad libitum. To induce impaired glucose tolerance, high-fat diet $(30 \%$ lard, $57 \%$ of total calories) was given for 3 weeks. Food intake was measured for 1 to 3 weeks in chow and high-fat diet fed rats. The F344 rats were cannulated in the right external jugular vein more than 5 days prior to the oral glucose tolerance test under sodium pentobarbital $(50 \mathrm{mg} / \mathrm{kg}$, i.p., Abbot Laboratories, North Chicago, IL, USA) anesthesia (15). For the in vitro assay of DPPIV activity, blood samples from SD rats were taken from the abdominal aorta and the plasma was separated and stored at $-80^{\circ} \mathrm{C}$.

\section{Oral glucose tolerance test}

The oral glucose tolerance test procedure has been described previously (15). Briefly, the rats were fasted over night and were transferred to the experimental room in each cage. The cannula of each animal was connected to a sampling tube, which was filled with saline solution to achieve a final concentration of $10 \mathrm{unit} / \mathrm{ml}$ heparin. After cage acclimation for 1 to $2 \mathrm{~h}$, NVP-DPP728 $(10 \mu \mathrm{mol} / \mathrm{kg}$ in $1 \mathrm{ml}$ water) was administered orally $30 \mathrm{~min}$ before the oral glucose challenge $(1 \mathrm{~g} / \mathrm{kg}$ in $5 \mathrm{ml}$ water, $\mathrm{t}=0 \mathrm{~min})$. Blood samples (about $300 \mu \mathrm{l}$ ) were obtained from the cannula before ( -5 and 0$)$ and at $3,5,10,15,20,30,45,60$, 75 and $90 \mathrm{~min}$ after the oral glucose challenge, and the blood glucose concentrations were analyzed immediately. After the final blood sampling ( $\mathrm{t}=90 \mathrm{~min})$, a blood sample (about $1 \mathrm{ml}$ ) was taken to assay plasma lipid levels. Blood samples were centrifuged $(1500 \times \mathrm{g}, 10 \mathrm{~min})$ at $4^{\circ} \mathrm{C}$. The plasma was stored at $-80^{\circ} \mathrm{C}$ until analysis of the plasma insulin concentration and DPP-IV activity.

\section{Plasma DPP-IV activity}

Plasma DPP-IV activity was determined by the cleavage rate of 7-amino-4-methylcoumarin (AMC; Sigma, St. Louis, MO, USA) from the synthetic substrate, $H$-glycyl-prolylAMC (Gly-Pro-AMC; Calbiochem-Novabiochem), as described previously $(15,25)$. In brief, $15 \mu \mathrm{l}$ of plasma was mixed with $135 \mu \mathrm{l}$ of $150 \mu \mathrm{M}$ Gly-Pro-AMC in assay buffer that was composed of $25 \mathrm{mM}$ tris(hydroxymethyl)aminomethane $\cdot \mathrm{HCl}(\mathrm{pH} 7.4), 140 \mathrm{mM} \mathrm{NaCl}, 10 \mathrm{mM} \mathrm{KCl}$, and $0.1 \%$ bovine serum albumin (Sigma). After incubation at room temperature, the fluorescence was determined using a spectrofluorometer (excitation at $380 \mathrm{~nm}$ and emission at $460 \mathrm{~nm})\left(\right.$ CytoFluor ${ }^{\mathrm{TM}} \mathrm{II}$; PerSeptive Biosystems, Framingham, MA, USA). DPP-IV activity in plasma was expressed as the amount of product (nmol) per minute per $\mathrm{ml}$. The compounds were added just before the incubation to determine their inhibitory effect in vitro.

\section{Blood glucose, plasma insulin and plasma lipid}

Blood glucose concentrations were measured by using a blood glucose analyzer (Antsense II $^{\circledR}$; Daikin, Osaka). Plasma insulin concentrations were measured by using an enzyme-linked immunosorbent assay (ELISA) using commercially available kits (Shibayagi, Gunma). Total cholesterol, triglycerides and phospholipids levels in plasma were measured by cholesterol oxidase-3,5-dimethoxy- $N$-ethyl- 
(2-hydroxy-3-sulfoproryl)-aniline sodium (DAOS), glycerol-3-phosphate oxidase-DAOS and choline oxidase-DAOS methods, respectively, using commercially available kits (Wako Pure Chemical Ind., Osaka) $(27,28)$.

\section{Statistical analyses}

Data were expressed as means \pm S.E.M. To determine the integrated glucose and insulin response to oral glucose challenge, incremental areas under the curves (AUC) of blood glucose and plasma insulin were calculated by the trapezoidal rule. Statistical analysis of the data was performed by two-way analysis of variance for time-course study and unpaired Student's $t$-test (two-tailed). Statistical significance was accepted at $P<0.05$.

\section{RESULTS}

\section{Plasma DPP-IV activity in rat strains}

Plasma DPP-IV activity was measured in F344 and SD rats. The fluorescence unit indicating the enzyme activity in F344 (Clea Japan) and SD (Charles River Japan) rat plasma increased proportionally in time-dependent manner (data not shown). The DPP-IV activity was $4.66 \pm 0.06$ and $5.54 \pm 0.41 \mathrm{nmol} \cdot \mathrm{min}^{-1} \cdot \mathrm{ml}^{-1}(\mathrm{n}=3)$, respectively. In contrast, no enzyme activity was detected in F344 (Charles River Japan) rat plasma. Thus, the deficiency of plasma DPP-IV activity was confirmed in Charles River Japan's F344 rats. All of the F344 (Charles River Japan) rats used in this study were verified to be deficient of plasma DPP-IV activity.
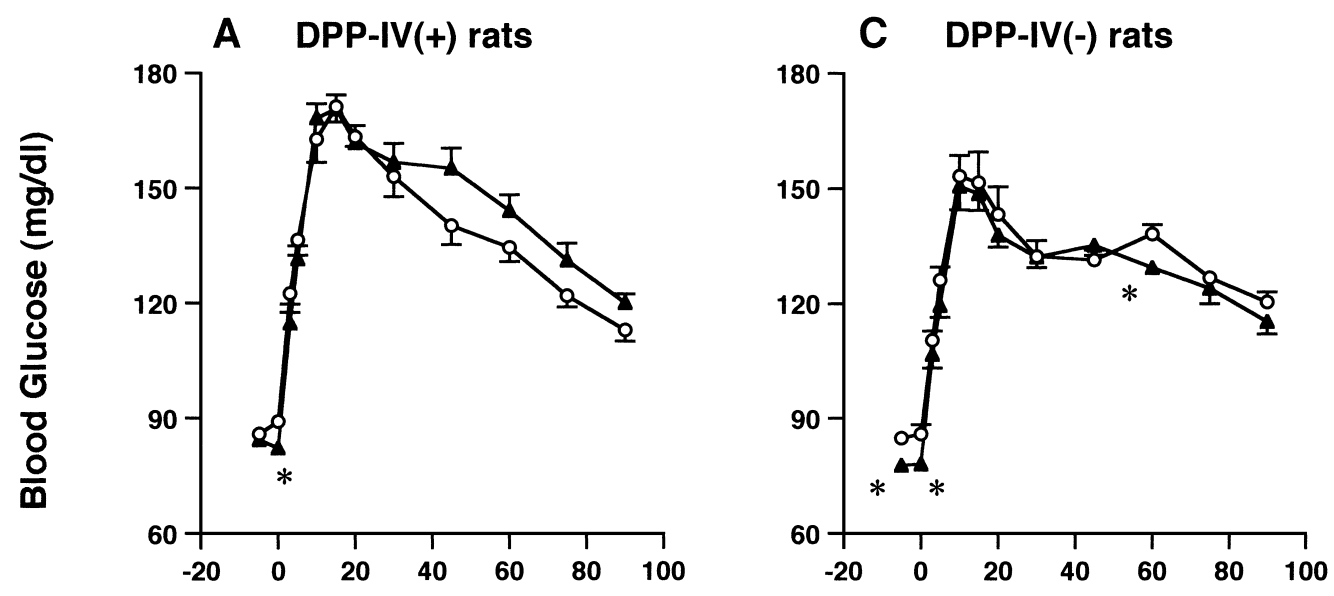

B
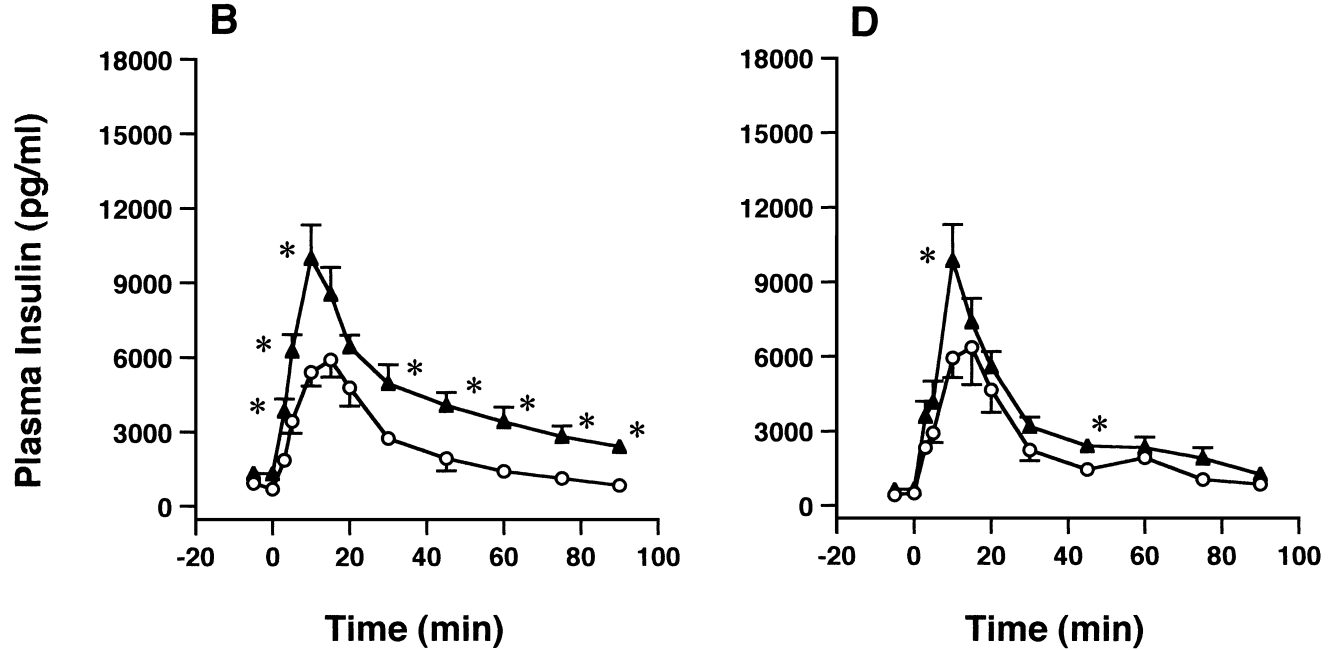

Fig. 1. Effects of the high-fat diet load on glucose metabolism in F344 rats. Dipeptidyl peptidase IV (DPP-IV) positive (+) $(\mathrm{A}$ and $\mathrm{B})$ and deficient $(-)(\mathrm{C}$ and $\mathrm{D})$ rats were given a standard chow (open circles) or the high-fat diet (closed triangles, $30 \%$ lard) for 3 weeks. Blood glucose (A and C) and plasma insulin (B and D) concentrations were determined during the oral glucose tolerance test $(1 \mathrm{~g} / \mathrm{kg}$, p.o., $\mathrm{t}=0 \mathrm{~min})$. Values are means \pm S.E.M. of $6-7$ animals. ${ }^{*} P<0.05$, significant difference from the standard chow group. 
Glucose metabolism in standard chow and high-fat diet-fed DPP-IV(+) and (-) rats

Feeding a high-fat diet for 3 weeks caused significantly impaired glucose tolerance in DPP-IV $(+)$ rats, even though the animals displayed a significantly increased insulin response (Fig. 1 and Table 1). In contrast, no marked significant change in glucose tolerance due to high-fat diet load was observed in DPP-IV(-) rats (Fig. 1). Although the peak level of insulin after the glucose challenge in the high-fat diet group was increased at $10 \mathrm{~min}$ in DPP-IV(-) rats, there was no significant difference in the integrated insulin response between the chow and high-fat diet groups (Fig. 1 and Table 1). Blood glucose concentrations in DPP$\mathrm{IV}(-)$ rats after glucose challenge were significantly lower than in DPP-IV $(+)$ rats under high-fat diet load conditions, although there was no difference in the integrated insulin response (Table 1). There were no significant differences in the food intake, body weight, total cholesterol, and triglyceride levels between the DPP-IV $(+)$ and $(-)$ control groups in both chow and high-fat diet-fed conditions, except that the plasma phospholipid levels in the high-fat diet-fed DPP-IV(-) rats was significantly lower than in the high-fat diet-fed DPP-IV $(+)$ rats (Table 2).

\section{Effect of NVP-DPP728 on the plasma DPP-IV activity}

NVP-DPP728 and a competitive substrate of DPP-IV, diprotin A, concentration-dependently inhibited DPP-IV activity (control value: $7.36 \mathrm{nmol} \cdot \mathrm{min}^{-1} \cdot \mathrm{ml}^{-1}, \mathrm{n}=3$ ) in vitro (data not shown). In this assay system, the 50\% inhibitory concentration $\left(\mathrm{IC}_{50}\right)$ of NVP-DPP728 and diprotin A was 0.024 and $8.6 \mu \mathrm{M}$, respectively. Oral administration of NVP-DPP728 $(10 \mu \mathrm{mol} / \mathrm{kg}) 30 \mathrm{~min}$ before the oral glucose challenge significantly and potently inhibited plasma DPP-IV activity throughout the oral glucose tolerance test in normal and high-fat diet-fed DPP-IV (+) rats (Fig. 2).

\section{Effect of NVP-DPP728 on the glucose metabolism}

Effects of oral administration of NVP-DPP728 on glucose metabolism were determined in standard chow and high-fat diet-fed rats. In chow-fed DPP-IV(+) rats, NVPDPP728 inhibited the increase of glucose concentrations in response to oral glucose challenge, associated with the

Table 1. Integrated glucose and insulin response to oral glucose challenge in normal and high-fat diet-fed F344 rats and the effects of NVPDPP728 on glucose metabolism

\begin{tabular}{llcccc}
\hline \multirow{2}{*}{ Conditions } & \multicolumn{2}{c}{ Glucose AUC } & \multicolumn{2}{c}{ Insulin AUC } \\
\cline { 3 - 6 } & & $\begin{array}{c}0-15 \mathrm{~min} \\
(\times 100 \mathrm{mg} / \mathrm{dl} \cdot 15 \mathrm{~min})\end{array}$ & $\begin{array}{c}15-60 \mathrm{~min} \\
(\times 100 \mathrm{mg} / \mathrm{dl} \cdot 45 \mathrm{~min})\end{array}$ & $\begin{array}{c}0-15 \mathrm{~min} \\
(\times 1000 \mathrm{pg} / \mathrm{ml} \cdot 15 \mathrm{~min})\end{array}$ & $\begin{array}{c}15-60 \mathrm{~min} \\
(\times 1000 \mathrm{pg} / \mathrm{ml} \cdot 45 \mathrm{~min})\end{array}$ \\
\hline DPP-IV(+) & Chow & $8.2 \pm 0.5$ & $26.6 \pm 1.4$ & $49.0 \pm 6.1$ & $93.1 \pm 18.5$ \\
DPP-IV(+) & Chow + NVP-DPP728 & $6.8 \pm 0.4$ & $17.3 \pm 1.2^{\mathrm{a}}$ & $103.2 \pm 12.0^{\mathrm{a}}$ & $68.1 \pm 18.4$ \\
DPP-IV(+) & High-fat & $9.1 \pm 0.2$ & $33.1 \pm 1.5^{\mathrm{b}}$ & $84.9 \pm 9.6^{\mathrm{b}}$ & $158.4 \pm 14.9^{\mathrm{b}}$ \\
DPP-IV(+) & High-fat + NVP-DPP728 & $6.2 \pm 0.4^{\mathrm{a}}$ & $20.4 \pm 1.3^{\mathrm{a}}$ & $112.0 \pm 15.6$ & $115.5 \pm 9.9^{\mathrm{a}}$ \\
DPP-IV(-) & Chow & $7.0 \pm 0.5$ & $22.5 \pm 1.9$ & $54.7 \pm 7.9$ & $91.7 \pm 14.6$ \\
DPP-IV(-) & Chow + NVP-DPP728 & $8.0 \pm 0.5$ & $20.9 \pm 1.4$ & $77.0 \pm 17.2$ & $73.1 \pm 13.0$ \\
DPP-IV(-) & High-fat & $7.6 \pm 0.2^{\mathrm{c}}$ & $25.4 \pm 1.0^{\mathrm{c}}$ & $82.4 \pm 10.0$ & $124.3 \pm 10.7$ \\
DPP-IV(-) & High-fat + NVP-DPP728 & $6.5 \pm 0.5$ & $23.3 \pm 2.1$ & $82.8 \pm 13.3$ & $143.4 \pm 15.7$ \\
\hline
\end{tabular}

Rats were given a standard chow or high-fat diet (30\% lard) for 3 weeks. Incremental areas under the curves (AUC) of blood glucose and plasma insulin were calculated by the trapezoidal rule. Values are means \pm S.E.M. of $6-7$ animals. ${ }^{a} P<0.05$, significant difference from the group fed the same diet in same animal substrain. ${ }^{b} P<0.05$, significant difference from the dipeptidyl peptidase IV (DPP-IV) $(+)$ fed chow diet group. ${ }^{c} P<0.05$, significant difference from the DPP-IV $(+)$ fed high-fat diet group.

Table 2. Feeding volume, body weight and plasma lipid levels in normal and high-fat diet-fed F344 rats

\begin{tabular}{llccccc}
\hline & Conditions & $\begin{array}{c}\text { Feeding volume } \\
(\mathrm{g} / \text { day })\end{array}$ & $\begin{array}{c}\text { Body weight } \\
(\mathrm{g})\end{array}$ & $\begin{array}{c}\text { Total cholesterol } \\
(\mathrm{mmol} / \mathrm{l})\end{array}$ & $\begin{array}{c}\text { Triglycerides } \\
(\mathrm{mmol} / \mathrm{l})\end{array}$ & $\begin{array}{c}\text { Phospholipids } \\
(\mathrm{mmol} / \mathrm{l})\end{array}$ \\
\hline DPP-IV(+) & Chow & $14.1 \pm 0.6$ & $253 \pm 2.5$ & $0.61 \pm 0.04$ & $0.24 \pm 0.01$ & $0.75 \pm 0.04$ \\
DPP-IV(+) & High-fat & $11.3 \pm 0.3$ & $279 \pm 3.5$ & $0.68 \pm 0.05$ & $0.36 \pm 0.06$ & $0.82 \pm 0.03$ \\
\hdashline DPP-IV(-) & Chow & $12.9 \pm 0.5$ & $262 \pm 6.0$ & $0.67 \pm 0.03$ & $0.20 \pm 0.02$ & $0.72 \pm 0.02$ \\
DPP-IV(-) & High-fat & $10.9 \pm 0.4$ & $280 \pm 3.7$ & $0.55 \pm 0.04$ & $0.23 \pm 0.02$ & $0.65 \pm 0.03^{\mathrm{a}}$ \\
\hline
\end{tabular}

Rats were given standard chow or high-fat diet (30\% lard) for 3 weeks. After the final blood sampling during an oral glucose tolerance test, a blood sample was taken to assay the lipid levels. Values are means \pm S.E.M. of $6-7$ animals. ${ }^{\text {a }} P<0.05$, significant difference from the high-fat diet-fed dipeptidyl peptidase IV (DPP-IV)(+) group. 


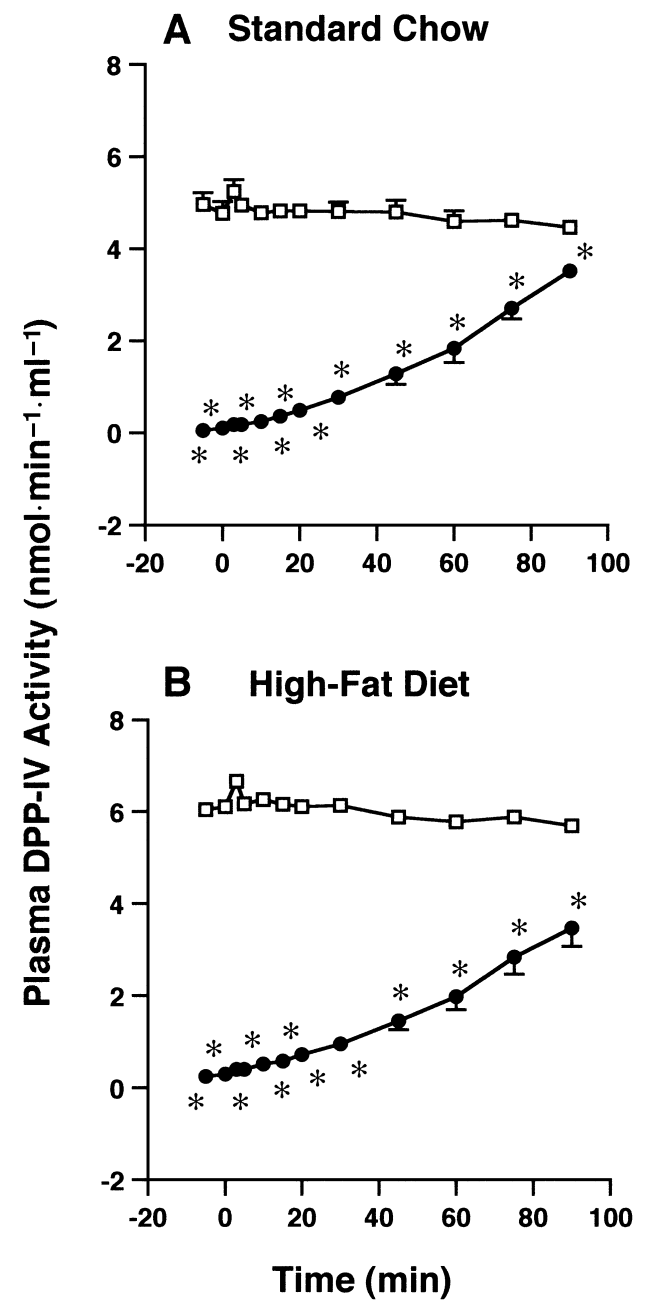

Fig. 2. Inhibition of plasma dipeptidyl peptidase IV (DPP-IV) activity by treatment with NVP-DPP728. Vehicle (open squares) or NVP-DPP728 (closed circles, $10 \mu \mathrm{mol} / \mathrm{kg}$, p.o.) was administered to standard chow (A) and high-fat diet (B)-fed DPP-IV(+) F344 rats $30 \mathrm{~min}$ before the glucose challenge $(1 \mathrm{~g} / \mathrm{kg}$, p.o., $\mathrm{t}=0)$. Blood samples were taken and plasma DPP-IV activity was measured. Values are means \pm S.E.M. of $6-7$ animals. ${ }^{*} P<0.05$, significant difference from the vehicle group.

enhanced early insulin response $(111 \%$ in Insulin AUC $(0-15 \mathrm{~min}))$, compared with the control group (Fig. 3 and Table 1). However, oral administration of NVP-DPP728 did not affect the glucose and insulin metabolism after glucose challenge in chow-fed DPP-IV(-) rats (Fig. 3 and Table 1).

Likewise, treatment with NVP-DPP728 significantly improved the impaired glucose tolerance induced by the high-fat diet in DPP-IV $(+)$ rats. The change in glucose response was $32 \%$ and $38 \%$ in Glucose AUC $(0-15 \mathrm{~min})$ and $(15-60 \mathrm{~min})$, respectively (Table 1$)$. The insulin level after glucose challenge was significantly elevated at $5 \mathrm{~min}$, although the integrated insulin response was not affected by NVP-DPP728 (Fig. 4). The improvement of glucose tolerance by NVP-DPP728 treatment was more potent and prolonged in the high-fat diet condition than in the chowdiet condition. On the other hand, there was no effect of NVP-DPP728 on the glucose and insulin metabolism after glucose challenge in the high-fat diet-fed DPP-IV(-) rats (Fig. 4).

\section{DISCUSSION}

Our experiments in the present study clearly demonstrated that feeding a high-fat diet caused markedly impaired glucose tolerance in DPP-IV $(+)$ rats. In contrast, no marked significant change in glucose metabolism was induced by the high-fat diet in DPP-IV $(-)$ rats. In addition, blood glucose concentrations after an oral glucose challenge in DPP-IV $(-)$ rats were significantly lower than in DPP-IV $(+)$ rats under high-fat diet load conditions. The DPP-IV inhibitor NVP-DPP728 potently inhibited plasma DPP-IV activity in vitro and in vivo. This led to improved glucose tolerance in chow and, especially, high-fat diet-fed DPP$\mathrm{IV}(+)$ rats. In contrast, there was no significant change in blood glucose and plasma insulin levels by the treatment of NVP-DPP728 in chow and high-fat diet-fed DPP-IV(-) rats.

Our results showed that DPP-IV(-) rats were resistant to the impaired glucose tolerance caused by high-fat diet load. Furthermore, the glucose tolerance after oral glucose challenge in DPP-IV $(-)$ rats was improved, compared with that in DPP-IV $(+)$ rats under both the normal chow and high-fat diet condition. Japanese F344 rats have been shown to lack the enzyme activity of DPP-IV (18). DPPIV $(-)$ rats contain mRNA transcripts for DPP-IV, but levels of DPP-IV protein are reduced due to translation of abnormal isoforms that fail to be processed to the biologically active mature glycosylated enzyme $(19,20)$. However, other peptidases such as aminopeptidase, neutral endopeptidase, and gamma-glutamyl transpeptidase are intact in DPP-IV(-) F344 rats (18). It has been demonstrated that mice lacking DPP-IV/CD26 show improved glucose tolerance associated with the enhanced insulin secretion and the increase in intact insulinotropic form of incretin levels (29). Since food intake and body weight were not different between DPP-IV $(+)$ and DPP-IV $(-)$ rats, these factors can not account for the difference in glucose tolerance between high-fat diet-fed DPP-IV $(+)$ and (-) rats. These results suggest that the resistance to metabolic deterioration in DPP-IV(-) rats exposed to a high-fat diet may result from the lack of the DPP-IV activity, and they indicate that chronic inhibition of DPP-IV might preserve glucose homeostasis through a protective effect on the development or progression of insulin resistance.

Oral administration of NVP-DPP728 significantly reduced glucose excursions after oral glucose challenge 

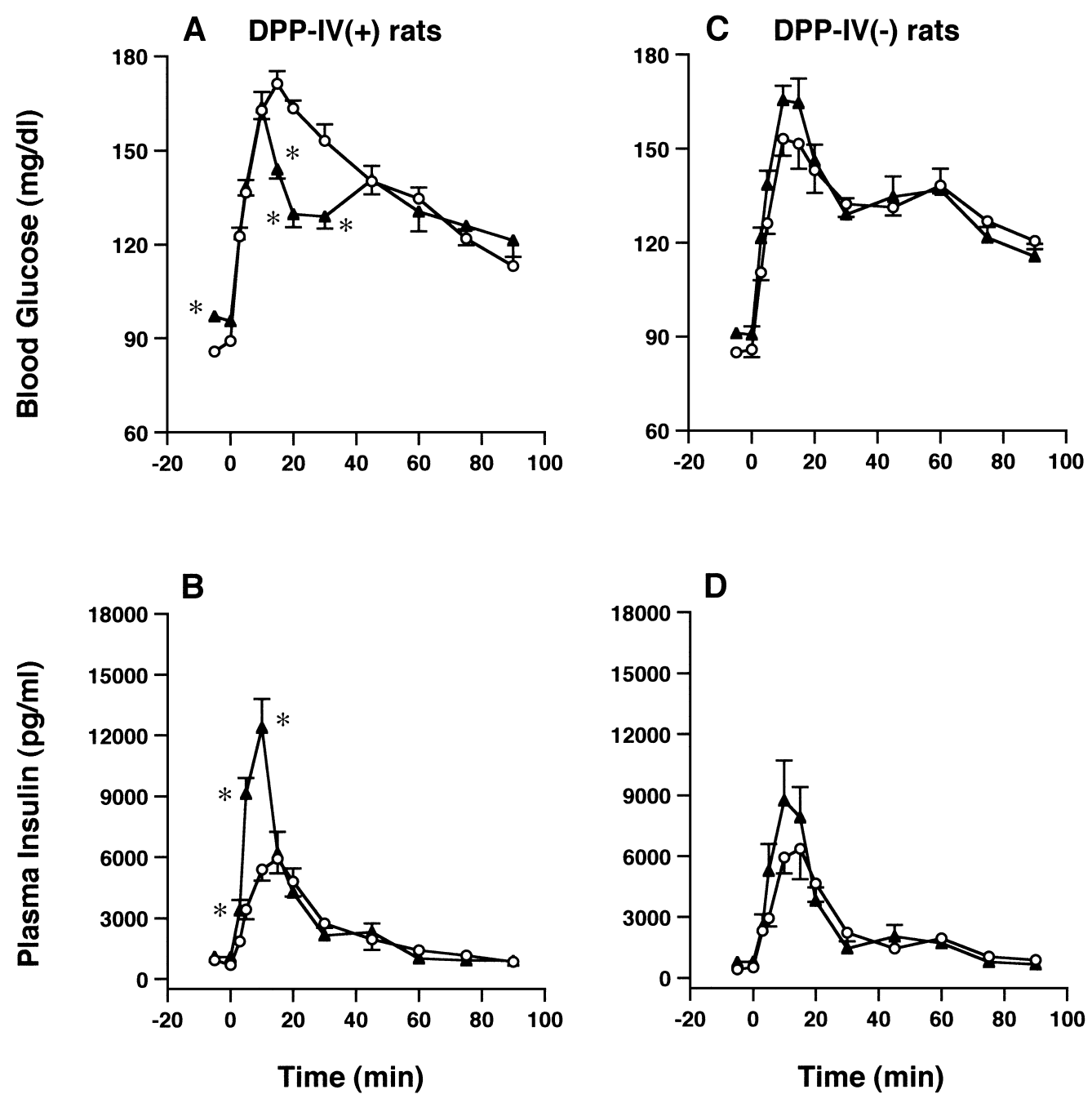

Fig. 3. Effects of NVP-DPP728 on glucose metabolism in a standard chow-fed F344 rats. Blood glucose (A and C) and plasma insulin (B and D) concentrations were determined during the oral glucose tolerance test $(1 \mathrm{~g} / \mathrm{kg}, \mathrm{p} . \mathrm{o} ., \mathrm{t}=0 \mathrm{~min})$ in dipeptidyl peptidase IV (DPP-IV) positive (+) (A and B) or deficient (-) (C and D) F344 rats. Vehicle (open circles) or NVP-DPP728 (closed triangles, $10 \mu \mathrm{mol} / \mathrm{kg}$, p.o.) was administered $30 \mathrm{~min}$ before the glucose challenge. Values are means \pm S.E.M. of $6-7$ animals. ${ }^{*} P<0.05$, significant difference from the vehicle group.

through the increase in early insulin release both in chow and high-fat diet fed DPP-IV(+) F344 rats. Our results were consistent with data indicating that acute oral administration of DPP-IV inhibitors augmented insulin responses to oral glucose challenge and enhanced glucose clearance in insulin resistant obese Zucker rats $(14,15)$. To support the notion that the observed action of NVP-DPP728 in DPP-IV $(+)$ rats was due to the inhibition of DPP-IV activity, we investigated the effect of NVP-DPP728 on the glucose tolerance in chow and high-fat diet fed DPP-IV(-) F344 rats. NVP-DPP728 did not affect the glucose-stimulated insulin release and glucose excursion in chow and high-fat diet fed DPP-IV(-) F344 rats. The glucose metabolism in chow and high-fat diet-fed DPP-IV(-) rats might mimic that in NVP-DPP728-treated normal and high-fat diet-fed DPP-IV $(+)$ rats, respectively. These results directly proved that the glucose-lowering action of NVP-DPP728 is due to the inhibition of plasma DPP-IV activity. The results further indicate that long-term treatment with DPPIV inhibitors may be effective to improve glucose tolerance and possibly diabetes without tachyphylaxis.

In the present study, the degree of enhancement of the integrated insulin response to glucose by NVP-DPP728 in high-fat diet-fed rats was lower than in normal rats. However, the reduction of glucose excursion was larger and prolonged in the high-fat diet group than that in the chowfed rat group. Although the precise reason for these results remains unclear, one possibility is that, since the high-fat diet load basically causes hyperinsulinemia in rats, the enhancement of insulinotropic action of increased endogenous incretin by NVP-DPP728 treatment was masked. Another potential explanation may involve the augmented 

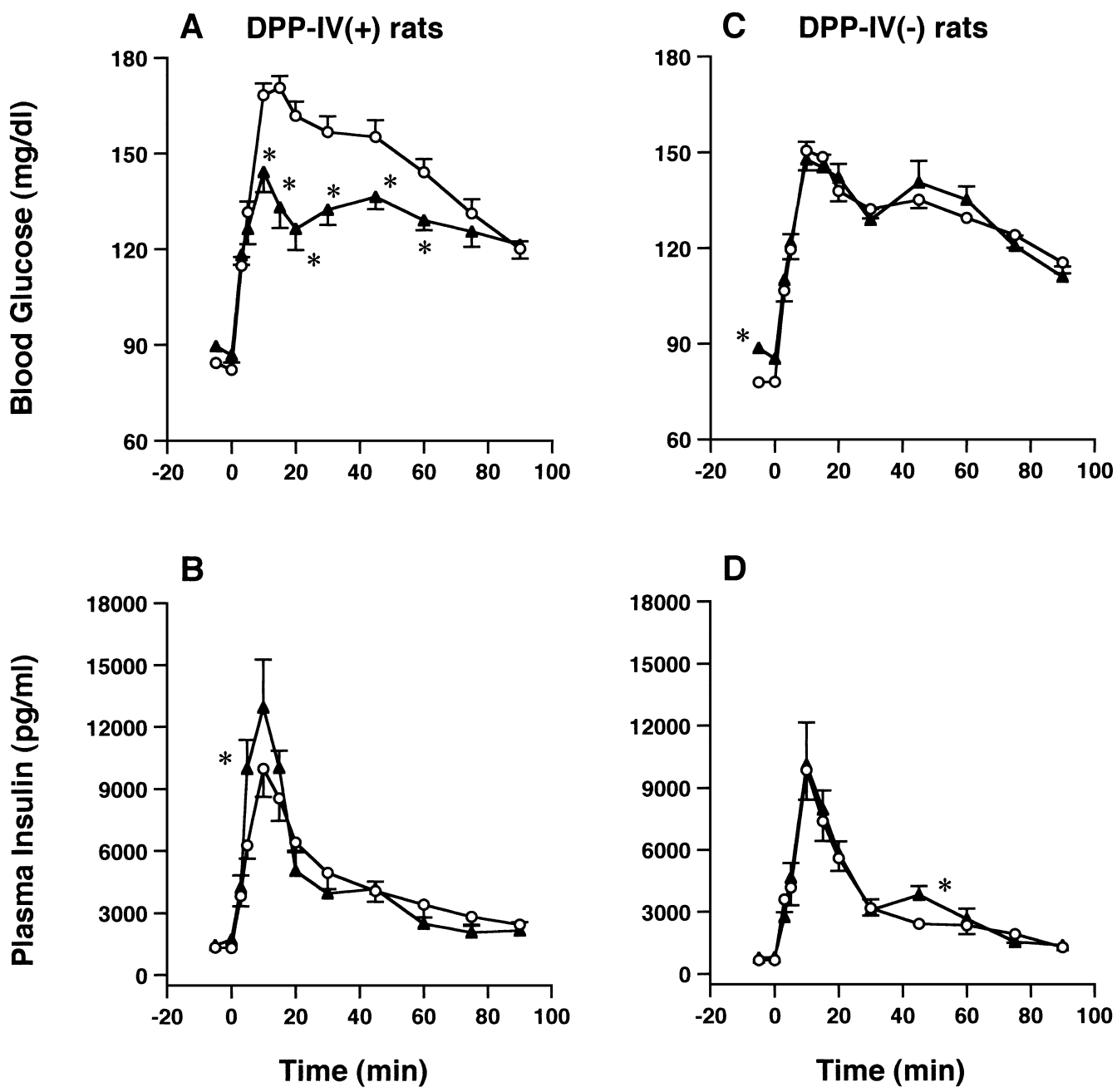

Fig. 4. Effects of NVP-DPP728 on glucose metabolism in high-fat diet-fed F344 rats. Dipeptidyl peptidase IV (DPP-IV) positive $(+)(\mathrm{A}$ and $\mathrm{B})$ or deficient $(-)$ (C and D) F344 rats were given the high-fat diet (30\% lard) for 3 weeks. Blood glucose $(\mathrm{A}$ and $\mathrm{C})$ and plasma insulin (B and D) concentrations were determined during the oral glucose tolerance test $(1 \mathrm{~g} / \mathrm{kg}$, p.o., $\mathrm{t}=0 \mathrm{~min}$ ). Vehicle (open circles) or NVP-DPP728 (closed triangles, $10 \mu \mathrm{mol} / \mathrm{kg}$, p.o.) was administered $30 \mathrm{~min}$ before the glucose challenge. Values are means \pm S.E.M. of $6-7$ animals. ${ }^{*} P<0.05$, significant difference from the vehicle group.

extra-pancreatic action of incretin, in which GLP-1 stimulated glucose utilization in peripheral tissue $(1-3)$. Recently, GLP-1 has increased insulin sensitivity dependent on the insulin dose presented in depancreatized dogs (30). Decreased glucagon levels, increased somatostatin levels, and decreased hepatic glucose production by augmented biological active GLP-1 may contribute to the action of NVP-DPP728 since the plasma glucagon level was stimulated by the high-fat diet load $(1-3)$.

It has been demonstrated that exogenously infused active GLP-1(7-36) amide was not degraded to N-terminally truncated inactive GLP-1 $(9-36)$ amide in DPP-IV $(-)$ rats (9). Moreover, although the plasma level of total GLP-1 (active and inactive) in DPP-IV(-) rats fed a normal chow is not different from that in normal chow fed DPP-IV $(+)$ rats (31), plasma active GLP-1 levels after intraduodenal glucose challenge were higher in the anesthetized DPP$\mathrm{IV}(-)$ rats than in the DPP-IV $(+)$ rats (32). DPP-IV inhibition by NVP-DPP728 increased plasma active GLP-1 concentrations during the oral glucose tolerance test in obese Zucker rats (15) and prevented inactivation of GLP1 in normal rats (33). These results suggest that the increase in active GLP-1 concentration might relate to the beneficial effects by the DPP-IV inhibition and the genetical depletion of DPP-IV activity. However, it has been demonstrated that DPP-IV inactivates a number of biological active peptides and factors such as glucose-dependent insulinotropic polypeptide (GIP), neuropeptide Y, peptide YY and peptide histidine methionine or its counterpart peptide histidine isoleucine, which are known to play a role in glucose metabolism $(1-3,16,34)$. Moreover, a recent finding demonstrated that DPP-IV inhibitor treatment improved glucose 
tolerance and enhanced glucose-stimulated insulin levels in mice lacking GLP-1 receptor, associated with the increase in plasma intact GIP concentrations (29). These results, the possible effect by NVP-DPP728 on the degradation of other peptides as well as GLP-1 may not only contribute to, but also counteract the beneficial effects of NVP-DPP728 on glucose tolerance. Further investigation will be needed to determine the contribution of incretins (GLP-1 and GIP) and other peptides on the DPP-IV inhibition-induced improvement of glucose tolerance.

In conclusion, our results suggest that the amelioration of glucose tolerance by treatment with NVP-DPP728 in DPP-IV $(+)$ rats was directly due to the inhibition of plasma DPP-IV activity, presumably via the subsequent increase in endogenous incretins. It is conceivable that the improved glucose tolerance was due to the enhancement of insulin secretion under the standard chow condition and due to both the moderate increased insulin secretion and possible increased insulin sensitivity under the high-fat diet condition. In addition, the difference in glucose tolerance between DPP-IV(+) and (-) rats especially under high-fat conditions indicates the possibility that plasma enzyme activity of DPP-IV limits the resistance to the development of impaired glucose tolerance. These data strongly support a therapeutic approach using a drug, which may potentiate the endogenous incretin action by long-term as well as acute inhibition of DPP-IV activity, to improve basal and prandial glycemic controls in patients with type 2 diabetes. As the first compound in a new chemical class, NVPDPP728 shows promise as a novel oral hypoglycemic agent that improves glycemia by increasing the activity of endogenous GLP-1 $(15,25,33)$. The present findings highlight the selectivity of effects of NVP-DPP728 and point to an intriguing possibility that long-term treatment with DPP-IV inhibitors may prevent metabolic deterioration in type 2 diabetes.

\section{Acknowledgments}

The authors thank Ms. K. Oda-Omae for excellent technical assistance and gratefully thank Dr. T. Okada and Dr. J. Ishikawa for giving us useful ideas and suggestions.

\section{REFERENCES}

1 Ahren B: Glucagon-like peptide-1 (GLP-1): a gut hormone of potential interest in the treatment of diabetes. Bioessays 20, $642-651$ (1998)

2 Drucker DJ: Glucagon-like peptides. Diabetes 47, 159-169 (1998)

3 Nauck MA: Is glucagon-like peptide 1 an incretin hormone? Diabetologia 42, 373 - 379 (1999)

4 Gutniak M, Orskov C, Holst JJ, Ahren B and Efendic S: Antidiabetogenic effect of glucagon-like peptide-1 (7-36) amide in normal subjects and patients with diabetes mellitus. N Engl J Med 326, 1316 - 1322 (1992)
5 Nauck MA, Kleine N, Orskov C, Holst JJ, Willms B and Creutzfeldt W: Normalization of fasting hyperglycaemia by exogenous glucagon-like peptide $1(7-36)$ amide in type 2 (non-insulin-dependent) diabetic patients. Diabetologia 36, 741 - 744 (1993)

6 Gutniak MK, Linde B, Holst JJ and Efendic S: Subcutaneous injection of the incretin hormone glucagon-like peptide 1 abolishes postprandial glycemia in NIDDM. Diabetes Care 17, 1039 - 1044 (1994)

7 Nauck MA, Wollschlaeger D, Werner J, Holst JJ, Oerskov C, Creutzfeldt W and Willms B: Effects of subcutaneous glucagonlike peptide 1 (GLP-1 [7 - 36 amide]) in patients with NIDDM. Diabetologia 39, 1546 - 1553 (1996)

8 Scrocchi LA, Brown TJ, MacLusky N, Brubaker PL, Auerbach $\mathrm{AB}$, Joyner AL and Drucker DJ: Glucose intolerance but normal satiety in mice with a null mutation in the glucagon-like peptide 1 receptor gene. Nat Med 2, 1254 - 1258 (1996)

9 Kieffer TJ, McIntosh CHS and Pederson RA: Degradation of glucose-dependent insulinotropic polypeptide and truncated glucagon-like peptide 1 in vitro and in vivo by dipeptidyl peptidase IV. Endocrinology 136, 3585 - 3597 (1995)

10 Deacon CF, Pridal L, Klarskov L, Olesen M and Holst JJ: Glucagon-like peptide 1 undergoes differential tissue-specific metabolism in the anesthetized pig. Am J Physiol 271, E458 - E464 (1996)

11 Mentlein R, Gallwitz B and Schmidt WE: Dipeptidyl-peptidase IV hydrolyzes gastric inhibitory polypeptide, glucagon-like peptide-1(7-36)amide, peptide histidine methionine and is responsible for their degradation in human serum. Eur J Biochem 214, 829 - 835 (1993)

12 Knudsen LB and Pridal L: Glucagon-like peptide-1-(9-36) amide is a major metabolite of glucagon-like peptide-1-(7-36) amide after in vivo administration to dogs, and it acts as an antagonist on the pancreatic receptor. Eur J Pharmacol 318, $429-435$ (1996)

13 Deacon CF, Hughes TE and Holst JJ: Dipeptidyl peptidase IV inhibition potentiates the insulinotropic effect of glucagon-like peptide 1 in the anesthetized pig. Diabetes 47, $764-769$ (1998)

14 Pederson RA, White HA, Schlenzig D, Pauly RP, McIntosh CHS and Demuth H-U: Improved glucose tolerance in Zucker fatty rats by oral administration of the dipeptidyl peptidase IV inhibitor isoleucine thiazolidide. Diabetes 47, 1253-1258 (1998)

15 Balkan B, Kwasnik L, Miserendino R, Holst JJ and Li X: Inhibition of dipeptidyl peptidase IV with NVP-DPP728 increases plasma GLP-1 (7 - 36 amide) concentrations and improves oral glucose tolerance in obese Zucker rats. Diabetologia 42, 1324 1331 (1999)

16 Holst JJ and Deacon CF: Perspectives in diabetes: inhibition of the activity of dipeptidyl-peptidase IV as a treatment for type 2 diabetes. Diabetes 47, 1663 - 1670 (1998)

17 Holst JJ, Deacon CF, Toft-Nielsen M-B and Bjerre-Kundsen L: On the treatment of diabetesmellitus with glucagon-like peptide-1. Ann N Y Acad Sci 865, 888 - 892 (1998)

18 Watanabe Y, Kojima T and Fujimoto Y: Deficiency of membrane-bound dipeptidyl aminopeptidase IV in a certain rat strain. Experientia 43, 400 - 401 (1987)

19 Thompson NL, Hixson DC, Callanan H, Panzica M, Flanagan D, Faris RA, Hong W, Hartel-Schenk S and Doyle D: A Fischer rat substrain deficient in dipeptidyl peptidase IV activity makes 
normal steady-state RNA levels and an altered protein. Use as a liver-cell transplantation model. Biochem J 273, 497-502 (1991)

20 Erickson RH, Suzuki Y, Sedlmayer A and Kim YS: Biosynthesis and degradation of altered immature forms of intestinal dipeptidyl peptidase IV in a rat strain lacking the enzyme. J Biol Chem 267, 21623 - 21629 (1992)

21 Iwaki-Egawa S, Watanabe Y and Fujimoto Y: Is CD26/dipeptidyl peptidase IV a really important molecule in T cell activation of a certain rat strain? Immunobiology 194, 429 - 442 (1995)

22 Rajvanshi P, Kerr A, Bhargava KK, Burk RD and Gupta S: Studies of liver repopulation using the dipeptidyl peptidase IVdeficient rat and other rodent recipients: cell size and structure relationships regulate capacity for increased transplanted hepatocyte mass in the liver lobule. Hepatology 23, 482 - 496 (1996)

23 Tanaka S, Murakami T, Horikawa H, Sugiura M, Kawashima K and Sugita T: Suppression of arthritis by the inhibitors of dipeptidyl peptidase IV. Int J Immunopharmacol 19, 15 - 24 (1997)

24 Morimoto C and Schlossman SF: The structure and function of CD26 in the T-cell immune response. Immunol Rev 161, 55 - 70 (1998)

25 Hughes TE, Mone MD, Russell ME, Welden SC and Villhauer EB: NVP-DPP728 (1-[2-[(5-cyanopyridin-2-yl)amino]ethylamino]acetyl-2-cyano-(S)-pyrrolidine), a slow-binding inhibitor of dipeptidyl peptidase IV. Biochemistry 38, 11597-11603 (1999)

26 Rahfeld J, Schierhorn M, Hartrodt B, Neubert K and Heins J: Are diprotin A (Ile-Pro-Ile) and diprotin B (Val-Pro-Leu) inhibitors or substrates of dipeptidyl peptidase IV? Biochim Biophys Acta 1076, 314-316 (1991)

27 Mitani H, Bandoh T, Ishikawa J, Kimura M, Totsuka T and
Hayashi S: Inhibitory effects of fluvastatin, a new HMG-CoA reductase inhibitor, on the increase in vascular ACE activity in cholesterol-fed rabbits. Br J Pharmacol 119, 1269 - 1275 (1996)

28 Mitani H, Bandoh T, Kimura M, Totsuka T and Hayashi S: Increased activity of vascular ACE related to atherosclerotic lesions in hyperlipidemic rabbits. Am J Physiol 271, H1065H1071 (1996)

29 Marguet D, Baggio L, Kobayashi T, Bernard AM, Pierres M, Nielsen PF, Ribel U, Watanabe T, Drucker DJ and Wagtmann N: Enhanced insulin secretion and improved glucose tolerance in mice lacking CD26. Proc Natl Acad Sci USA 97, 6874-6879 (2000)

30 Sandhu H, Wiesenthal SR, MacDonald PE, McCall RH, Tchipashvili V, Rashid S, Satkunarajah M, Irwin DM, Shi ZQ, Brubaker PL, Wheeler MB, Vranic M, Efendic S and Giacca A: Glucagon-like peptide 1 increases insulin sensitivity in depancreatized dogs. Diabetes 48, 1045 - 1053 (1999)

31 Pederson RA, Kieffer TJ, Pauly R, Kofod H, Kwong J and McIntosh CHS: The enteroinsular axis in dipeptidyl peptidase IV-negative rats. Metabolism 45, 1335 - 1341 (1996)

32 Nagakura T, Yasuda N, Yamazaki K, Ikuta H, Yoshikawa S, Asano $\mathrm{O}$ and Tanaka $\mathrm{I}$ : Improved glucose tolerance via enhanced glucose-dependent insulin secretion in dipeptidyl peptidase IV-deficient Fischer rats. Biochem Biophys Res Commun 284, 501 - 506 (2001)

33 Hughes TE, Balkan B and Villhauer EB: NVP-DPP728, a novel, orally active dipeptidyl peptidase IV (DPP-IV) inhibitor, prevents glucagon-like peptide-1 (GLP-1) inactivation in rats (Abstract). Diabetes 48, Suppl 1, A21 (1999)

34 Mentlein R: Dipeptidyl-peptidase IV (CD26) - role in the inactivation of regulatory peptides. Regul Pept 85, 9 - 24 (1999) 